\title{
A markerless gene deletion and integration system for Thermoanaerobacter ethanolicus
}

Xiongjun Shao ${ }^{1,2}$, Jilai Zhou ${ }^{1,2}$, Daniel G. Olson ${ }^{1,2}$ and Lee R. Lynd ${ }^{1,2^{*}}$

\begin{abstract}
Background: Thermoanaerobacter ethanolicus produces a considerable amount of ethanol from a range of carbohydrates and is an attractive candidate for applications in bioconversion processes. A genetic system with reusable selective markers would be useful for deleting acid production pathways as well as other genetic modifications.

Results: The thymidine kinase ( $t d k)$ gene was deleted from T. ethanolicus JW200 to allow it to be used as a selectable marker, resulting in strain X20. Deletion of the $t d k$ gene reduced growth rate by $20 \%$; however, this could be reversed by reintroducing the $t d k$ gene (strain $\mathrm{X} 20 \mathrm{C}$ ). The $t d k$ and high-temperature kanamycin ( $h t k)$ markers were tested by using them to delete lactate dehydrogenase (Idh). During positive selection of Idh knockouts in strain X20 on kanamycin agar plates, six out of seven picked colonies were verified transformants. Deletion of $/ d h$ reduced lactic acid production by $90 \%$. The $t d k$ and 5-fluoro-2'-deoxyuridine (FUDR) combination worked reliably as demonstrated by successful tdk removal in all 21 colonies tested.

Conclusion: A gene deletion and integration system with reusable markers has been developed for Thermoanaerobacter ethanolicus JW200 with positive selection on kanamycin and negative selection on FUDR. Gene deletion was demonstrated by Idh gene deletion and gene integration was demonstrated by re-integration of the tdk gene. Transformation via a natural competence protocol could use DNA PCR products amplified directly from Gibson Assembly mixture for efficient genetic modification.
\end{abstract}

Keywords: Thermoanaerobacter ethanolicus, Gene deletion, Gene integration, Marker removal, Clean knockout, tdk, FUDR, Natural competence

\section{Background}

Plant biomass is the only foreseeable sustainable source of organic fuels, chemicals, and materials available to humanity [1]. Lignocellulosic biomass is particularly attractive in this context because of its widespread availability and low cost [2]. Thermoanaerobacter ethanolicus is a gram-positive, anaerobic thermophile which can produce a considerable amount of ethanol from a wide range of polymeric and soluble carbohydrates [3] and is of interest for bioconversion processes [4].

\footnotetext{
*Correspondence: lee.lynd@dartmouth.edu

114 Engineering Drive, Thayer School of Engineering, Dartmouth College, Hanover, NH 03755, USA

Full list of author information is available at the end of the article
}

For commercial application of Thermoanaerobacter or Thermoanaerobacterium species, genetic engineering is likely necessary to remove by-product formation and increase the yield of final product [5-7]. Electrotransformation has been reported for Thermoanaerobacter ethanolicus JW200 with low transformation efficiency [8]. The discovery of natural competence and relatively hightransformation efficiency for T. ethanolicus JW200 and many other Thermoanaerobacter or Thermoanaerobacterium species has greatly reduced the complexity of DNA manipulation in these bacteria [9]. Metabolic engineering involving multiple genetic manipulations requires either multiple selective markers, which are not described for $T$. ethanolicus, or removable selective markers. Two marker removal systems have been developed for 
Thermoanaerobacterium saccharolyticum. One is based on the $\operatorname{pyr} F$ gene conferring sensitivity to 5 -fluoroorotic acid (5-FOA). The other is based on the pta-ack gene cassette conferring sensitivity to haloacetate [10]. Both of these systems have certain disadvantages. The pyrF system requires media without uracil, and the $p t a-a c k$ system requires a $\mathrm{pH}$ of around 5 . Therefore, neither of these systems is suitable for $T$. ethanolicus, which requires yeast extract for growth and does not grow well at $\mathrm{pH} 5$.

Two additional marker removal systems have been developed in thermophilic bacterium Clostridium thermocellum: Hypoxanthine phosphoribosyl transferase (Hpt) encoded by $h p t$ which confers sensitivity to 8 -azahypoxanthine $(8 \mathrm{AZH})$ and thymidine kinase (Tdk) encoded by $t d k$ which confers sensitivity to 5 -fluoro-2'deoxyuridine (FUDR) [11]. The hpt gene has also been developed as a negative selection marker for Archaea [12]. The $t d k$ gene has a key role in the synthesis of DNA and in cell division because it is part of the pathway used to salvage thymidine from degraded DNA $[13,14]$. Tdk converts FUDR to fluoro-dUMP (F-dUMP), which inhibits ThyA. ThyA catalyzes an essential reaction, converting dUMP to dTMP in nucleic acid metabolism. The $t d k$ marker has been used for counter-selection in a variety of eukaryotic organisms [15-17], although its use in prokaryotes is rare (the only report to date being C. thermocellum). The $t d k$ gene used in C. thermocellum is originally from T. saccharolyticum, indicating this strategy may also work in other thermophilic microorganisms, especially Thermoanaerobacter and Thermoanaerobacterium genus.

In this paper, we sought to develop a marker recycling system for T. ethanolicus, based on the positive Kanamycin selection using the $h t k$ marker, followed by negative FUDR selection using the $t d k$ marker, which could function in the presence of yeast extract and at a neutral $\mathrm{pH}$.

\section{Results}

Genetic basis of FUDR selection in T. ethanolicus JW200

Unlike $C$. thermocellum, $T$. ethanolicus has a putative $t d k$ gene that encodes thymidine kinase, Teth_0051. Protein BLAST indicated that the enzyme encoded by Teth_0051 had $82 \%$ identity to that of T. saccharolyticum used in $C$. thermocellum (Tsac_0324). We also found a putative gene (Teth_1237) encoding ThyA, which would be inhibited by F-dUMP.

\section{Deletion of $t d k$ in wild-type strain}

To create a background strain from which an antibiotic resistance marker can be removed via negative selection, wild-type T. ethanolicus JW200 was transformed with PCR products from plasmid pTE_tdk (Fig. 1b) to delete the $t d k$ gene. Upstream and downstream homologous recombination fragments (800-1100 bp) were selected without any overlap with the $t d k$ gene and its rbs region as illustrated in Fig. 2a. Recombination events resulting in the deletion of $t d k$ are shown in Fig. 2b. No colonies appeared on the agar plates with $50 \mathrm{mg} / \mathrm{L}$ FUDR when inoculated with the wild-type. Seven colonies on plates inoculated with transformed cells were picked and analyzed by colony PCR. The resulting PCR products were analyzed by DNA gel electrophoresis, which is shown in Fig. 3. All seven colonies picked from FUDR plates displayed a band size of $2.0 \mathrm{~kb}$ compared to a band size of $2.7 \mathrm{~kb}$ for the wild-type control. Confirmed by sequencing, colony 6 , denoted strain X20, was chosen as the parent strain for subsequent gene deletion and integration experiments.

\section{Gene deletion}

To demonstrate gene deletion, the $l d h$ gene was deleted in the $t d k$ deletion background (strain X20). Strain X20 was transformed with PCR products from plasmid pTE_ldh (Fig. 1c). Upstream, internal, and downstream homologous recombination fragments (800-1100 bp) were selected according to primer design for PCR amplification (Fig. 2a). Recombination events resulting in the deletion of $l d h$ is shown in Fig. 2c. Seven colonies were picked on CTFUD agar plates with $400 \mathrm{mg} / \mathrm{L}$ kanamycin sulfate plated with transformed culture. No colonies were observed on the kanamycin agar plate for the X20 control strain. The picked colonies were analyzed by colony PCR with the X20 strain as control. The DNA gel electrophoresis diagram loaded with colony PCR products is shown in Fig. 4a. Six out of seven picked colonies were confirmed to have the correct DNA integration, with a band size of $4.9 \mathrm{~kb}$ compared to a band size of $1.7 \mathrm{~kb}$ for the X20 control strain. At this stage, the $l d h$ gene had been disrupted by the $h t k$ - $t d k$ cassette. The next step was to remove the $h t k-t d k$ cassette by counter-selection with FUDR.

Colony 6 was chosen and plated on CTFUD agar plates with $50 \mathrm{mg} / \mathrm{L}$ FUDR to select for cells without a functional $t d k$ marker. It was expected that the $t d k$ and $h t k$ markers would be lost simultaneously. Seven colonies were picked from the FUDR agar plate. The picked colonies were analyzed by colony PCR with the X20 strain as control. The DNA gel electrophoresis diagram is shown in Fig. 4b. All seven colonies were confirmed to have the correct DNA size, with a band size of $2.0 \mathrm{~kb}$ compared to a band size of $2.6 \mathrm{~kb}$ for the X20 control strain. Colonies 2 and 7 were confirmed by sequencing and used for subsequent fermentation characterization.

\section{Gene integration}

To demonstrate gene integration, the $t d k$ gene was inserted back into strain X20. PCR products from plasmid pTE_tdk(com) (Fig. 1d) were transformed into strain $\mathrm{X} 20$. Recombination events resulting in the insertion 

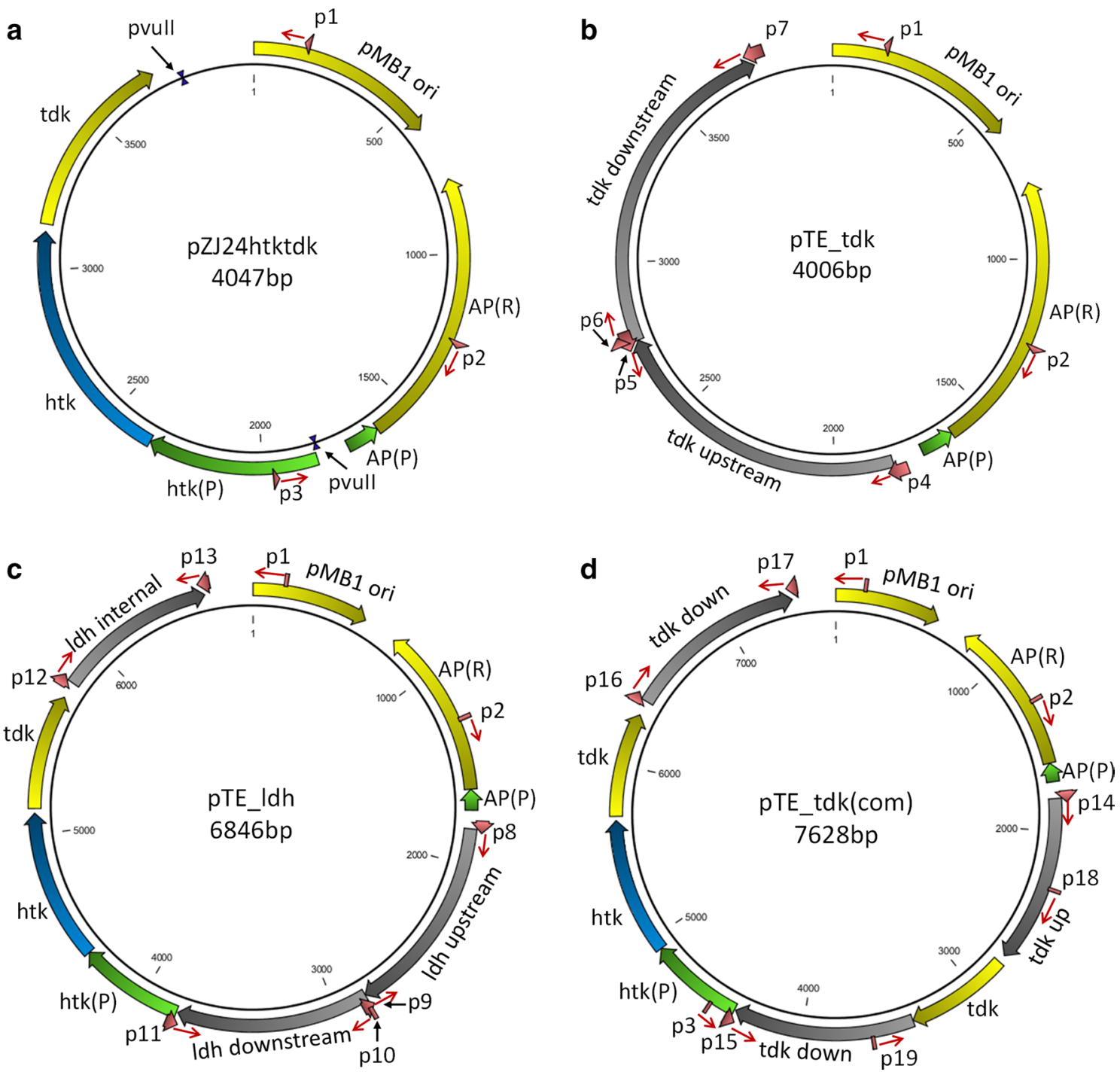

Fig. 1 Plasmid maps for gene deletion and integration: a backbone plasmid, b plasmid for $t d k$ deletion in wild-type, $\mathbf{c}$ plasmid for Idh deletion in stain X20, d plasmid for tdk integration in strain X20. Red: primers; green: promoters; yellow: coding region or origin of replication; blue: coding region for htk; gray: region for homologous recombination. AP ampicillin; up upstream; down downstream; ori origin; $R$ resistance; $P$ promoter; red arrow primer direction

of $t d k$ into strain X20 is shown in Fig. 2d. No colonies appeared on the kanamycin agar plate for the X20 control strain. Seven colonies were picked on CTFUD agar plates with $400 \mathrm{mg} / \mathrm{L}$ kanamycin sulfate. The picked colonies were analyzed by colony PCR (Fig. 5a). Four out of seven picked colonies were confirmed to have correct DNA integration, with a band size of $2.8 \mathrm{~kb}$ compared to the expected no band for the X20 control strain. At this point, the strain had two copies of the $t d k$ marker on the chromosome: one at its native locus and one downstream of the $h t k$ gene.

To eliminate the second copy of the $t d k$ gene, cells were plated at a low concentration of FUDR. Concentrations of 0 and $5 \mathrm{mg} / \mathrm{L}$ were tested. Seven colonies were picked from the FUDR agar plate. The picked colonies were analyzed by colony PCR (Fig. 5b). All seven colonies appeared to have correct deletions, with a band size of $1.3 \mathrm{~kb}$ compared to a band size of $0.7 \mathrm{~kb}$ for the X20 control strain. Four colonies were chosen $(\mathrm{C} 1, \mathrm{C} 2, \mathrm{C} 3$, and C7) for sequencing and all were confirmed to have the same sequence as the wild-type strain. Colonies 2 and 7 were selected for fermentation characterization.

\section{Comparison of fermentation profile}

Fermentation products were measured for the engineered strains and the wild-type strain (Table 1). Two 
a
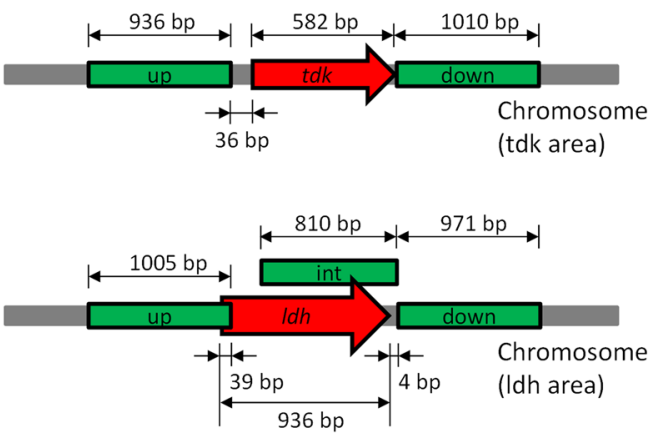

C

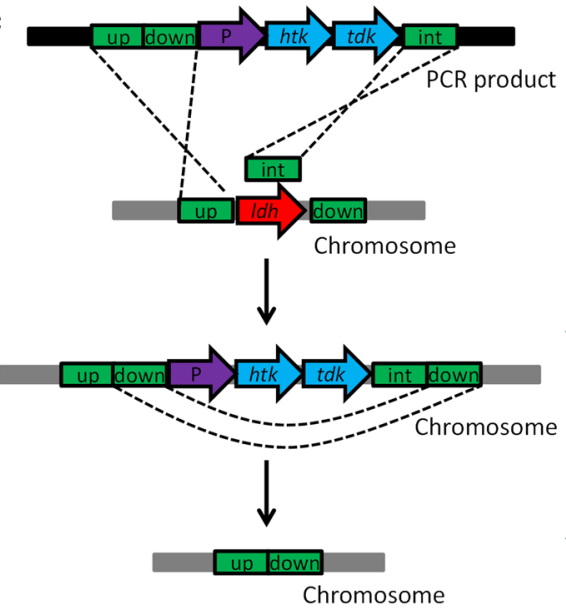

b

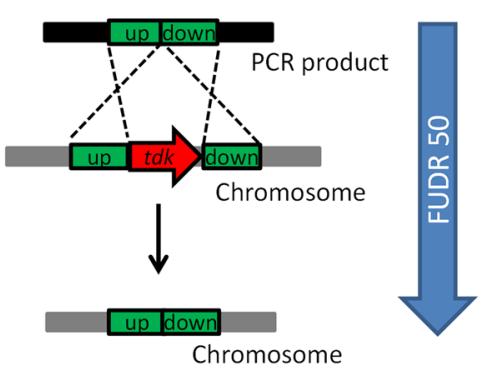

d

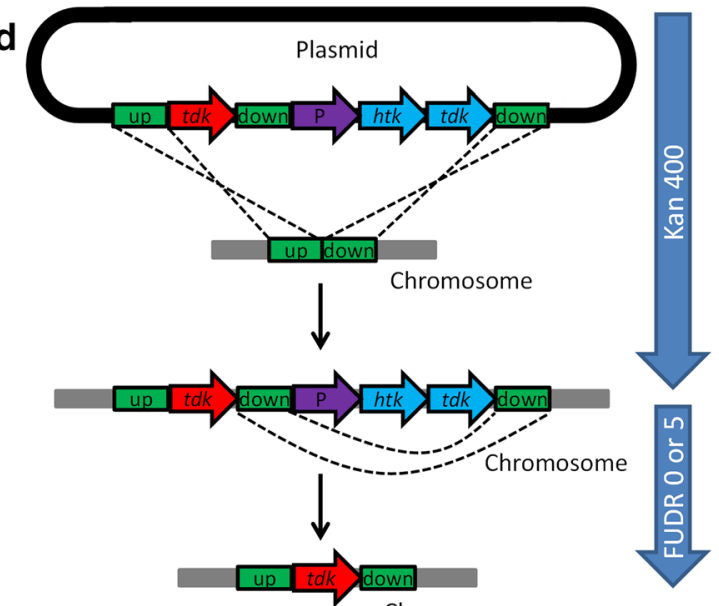

Chromosome

Fig. 2 Design of homologous recombination fragments (a) recombination events for deleting $t d k$ in wild-type strain (b) recombination events for deleting $/ d h$ in X20 (c) and recombination events for inserting $t d k$ into X20 (d) P promoter; up upstream; down downstream; int internal

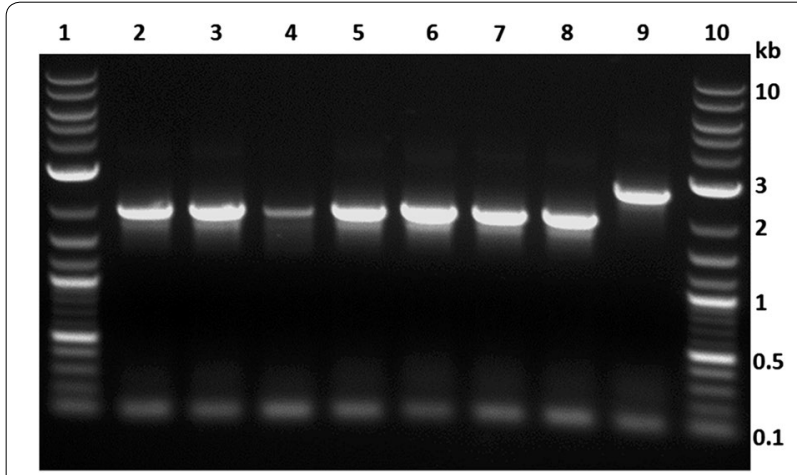

Fig. 3 Gel confirmation for tdk deletion in wild-type (WT) strain. Lanes 1 and 10: 2-log DNA ladder; lanes 2-8: seven picked colonies; lane 9:WT control wild-type stocks were cultured, each with five replicates. Two colonies were analyzed for X20 and X32, respectively. All seven colonies picked for X20C were cultured. The two wild-type stocks had quite different fermentation product profiles, with the $\mathrm{WT}^{*}$ stock having much higher organic acid production and lower ethanol yield. Both stocks were confirmed by $16 \mathrm{~S}$ sequencing. The reason for the variation in fermentation product profile, also observed by the original researchers [3], was unknown. Compared to the WT strain, the X20 strain had about 4-5\% decrease in carbon recovery from measured fermentation products $\left(R_{c}\right)$ and a $20-25 \%$ decrease in specific growth rate $(\mu)$. Deletion of $l d h$ (strain X32) reduced lactate production by $90 \%$ and increased ethanol and acetate production slightly. Compared to the X20 parent strain, there was about $4 \%$ decrease in carbon recovery and $10 \%$ decrease in specific growth rate. Reintroduction of the $t d k$ gene in the X20 strain restored the growth rate and fermentation product profile observed in the WT strain.

\section{Discussion}

A markerless gene deletion and integration system has been developed for T. ethanolicus JW200 that combines the $h t k$ positive selection marker and the $t d k$ negative selection marker. Of 14 colonies picked from kanamycin selection plates, four were false positives. Of 21 colonies 


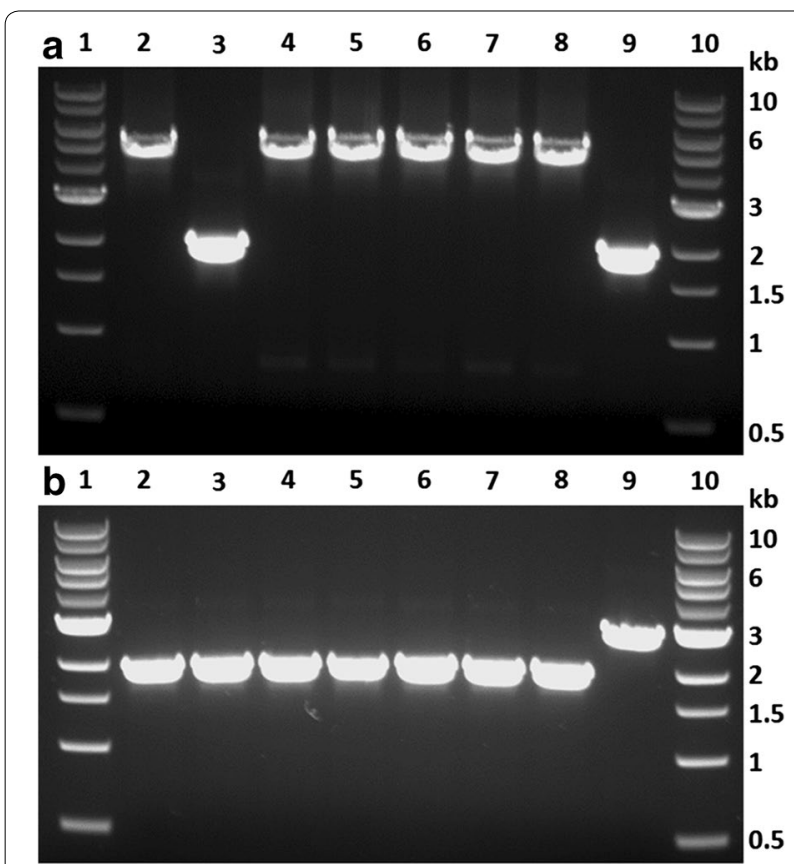

Fig. 4 Gel confirmation for Idh deletion in X20. a Lanes 1 and 10: $1 \mathrm{~kb}$ DNA ladder; lanes 2-8: seven colonies picked from kanamycin agar plate; lane 9: X20 control. b Lanes 1 and 10: 1 kb DNA ladder; lanes 2-8: seven colonies picked from FUDR agar plate; lane 9: X20 control

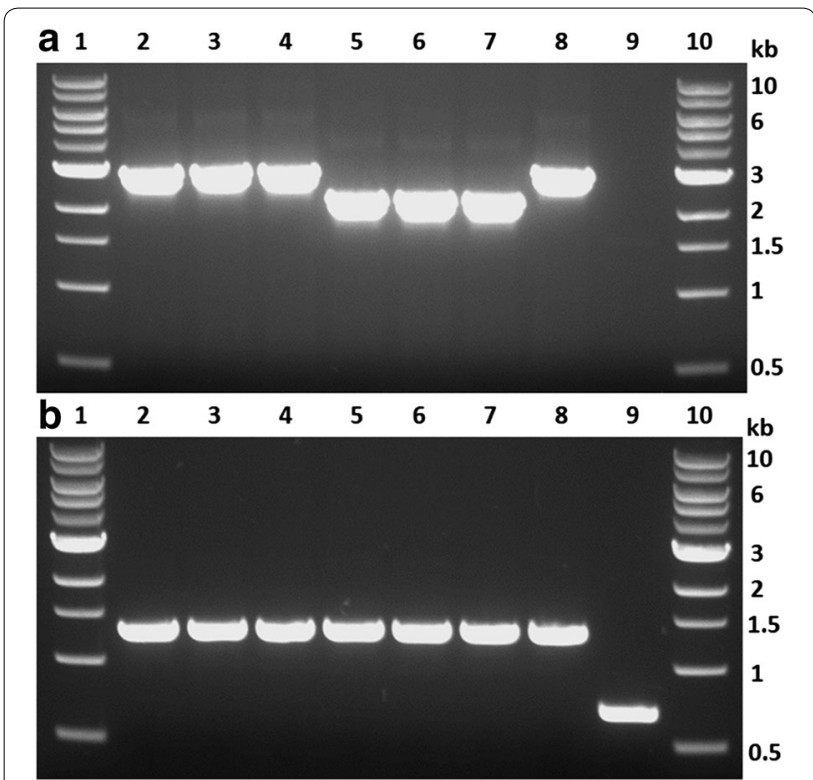

Fig. 5 Gel confirmation for integration of $t d k$ in X20. a Lanes 1 and 10 : 1 kb DNA ladder; lanes 2-8: seven colonies picked from kanamycin agar plate; lane 9: X20 control. b Lanes 1 and 10: 1 kb DNA ladder; lanes 2-8: seven colonies picked from FUDR agar plate; lane 9: X20 control

picked from FUDR selection plates, none were false positives. Four out of seven colonies were positive on kanamycin selection for $t d k$ integration, while six out of seven colonies were positive on kanamycin selection for $l d h$ deletion. This lower success rate for $t d k$ integration was probably caused by the fact that there were two identical $t d k$ downstream regions in the plasmid.

Deletion of $t d k$ resulted in reduced cell growth rate (20\%) and carbon recovery (calculated from measured fermentation products). The reason why this deletion increased ethanol yield is unknown. Reintroduction of $t d k$ restored cell growth rate to that of wild-type, but ethanol yield remained as that of $t d k$ deletion strain. Overexpression of $t d k$ is expected to be unfavorable based on the observation that homologous recombination occurred spontaneously to remove the DNA between the two downstream regions.

The discovery of natural competence in T. ethanolicus has greatly increased the convenience of its genetic modification. Transformation efficiency of T. ethanolicus was the highest among several species reported in a recent study [9]. This high-transformation efficiency makes it possible to transform with PCR products amplified directly from Gibson Assembly products, further simplifying genetic manipulation. The length of DNA fragments for homologous recombination had a large impact on the recombination frequency. We recommend using a length of $800-1000 \mathrm{bp}$ based on the experience of genetic manipulations in T. ethanolicus and C. thermocellum, and further that 400-500 bp be left on both ends of the entire DNA fragment when using PCR products instead of circular plasmids. This approach, also reported by Hashimoto et al. [18], would protect the regions of homologous recombination from degradation before they reached recombination.

If the target gene for deletion is relatively small (e.g., $<800 \mathrm{bp}$ ), an internal fragment might be too short for efficient homologous recombination. In this situation, a potential alternative gene deletion approach is to use upstream and downstream homologous recombination fragments and perform transformation twice. The first transformation uses DNA PCR products with antibiotic marker and $t d k$ gene between the two upstream and downstream fragments followed by selection on agar plates with the antibiotic. Using a positive colony from the first step, the second transformation uses DNA PCR products with the two upstream and downstream fragments connected directly followed by selection on agar plates with FUDR.

The gene deletion and integration system reported here does not need manipulation of medium components and other growth conditions such as medium $\mathrm{pH}$, thus greatly reducing complexity of genetic modification compared to previously described protocols [10]. It is potentially applicable to other species as long as they can express $t d k$, are sensitive to FUDR selection, have an antibiotic 
Table 1 Fermentation profile of strains

\begin{tabular}{lllllll}
\hline Strain & Lactate, $\mathbf{g} / \mathbf{L}$ & Acetate, $\mathbf{g} / \mathbf{L}$ & Ethanol, $\mathbf{g} / \mathbf{L}$ & $\boldsymbol{R}_{\mathbf{c}}$ & $\boldsymbol{Y}_{\text {eth }}$ & $\boldsymbol{\mu}_{\mathbf{,}} \mathbf{h}^{\mathbf{1}}$ \\
\hline WT & $0.604 \pm 0.024$ & $0.271 \pm 0.029$ & $1.655 \pm 0.008$ & $0.950 \pm 0.009$ & $0.370 \pm 0.002$ & $0.292 \pm 0.009$ \\
WT $^{\mathrm{a}}$ & $1.690 \pm 0.038$ & $0.545 \pm 0.028$ & $0.880 \pm 0.039$ & $0.945 \pm 0.002$ & $0.197 \pm 0.009$ & $0.272 \pm 0.011$ \\
X20 & $0.654 \pm 0.009$ & $0.298 \pm 0.003$ & $1.493 \pm 0.000$ & $0.900 \pm 0.001$ & $0.334 \pm 0.000$ & $0.223 \pm 0.010$ \\
X32 & $0.068 \pm 0.003$ & $0.322 \pm 0.003$ & $1.695 \pm 0.018$ & $0.866 \pm 0.010$ & $0.379 \pm 0.004$ & $0.196 \pm 0.002$ \\
X20C & $0.552 \pm 0.015$ & $0.267 \pm 0.009$ & $1.649 \pm 0.021$ & $0.934 \pm 0.008$ & $0.368 \pm 0.005$ & $0.285 \pm 0.003$ \\
\hline
\end{tabular}

a Wild-type of a different stock

marker for positive selection, and are able to perform homologous recombination.

\section{Conclusion}

A markerless gene deletion and integration system has been developed for Thermoanaerobacter ethanolicus JW200 with positive selection on kanamycin and negative selection on FUDR. Gene deletion was demonstrated by $l d h$ gene deletion and gene integration was demonstrated by re-integration of $t d k$ gene. Transformation could use DNA PCR products amplified directly from Gibson Assembly mixture for efficient genetic modification. Deletion of $l d h$ gene nearly removed lactic acid production. Re-integration of $t d k$ gene was desirable because its removal resulted in significant drop of specific growth rate $(20 \%)$.

\section{Methods}

Strains and culturing conditions

Thermoanaerobacter ethanolicus strain ATCC 31550 (JW200, DSM 2246) was obtained from ATCC. The strain was cultured in CTFUD medium [19] with or without $0.8 \%(\mathrm{w} / \mathrm{v})$ agar with an initial $\mathrm{pH}$ of 7 at $65^{\circ} \mathrm{C}$ in an anaerobic chamber (Coy Laboratory Products, Grass Lake, MI).

\section{Construction of vectors}

DNA fragments were amplified by PCR using the primers listed in Table 2. The PCR products were gel-purified. All deletion vectors used in this study were derived from plasmid pZJ24 which was created by replacing the erythromycin gene with a kanamycin gene from plasmid pZJ23 [20]. The kanamycin resistance gene in pZJ24 was replaced with a high-temperature kanamycin resistance gene $(h t k)$. The $h t k$ marker was developed by Hoseki et al. for kanamycin selection at temperatures up to $72{ }^{\circ} \mathrm{C}$ [21]. The thymidine kinase gene $(t d k)$ with ribosome-binding site from T. ethanolicus was added downstream of the $h t k$ gene to generate the backbone plasmid pZJ24htktdk as shown in Fig. 1a. The backbone plasmid was digested by PvuII and column purified. The upstream, internal, and downstream regions of gene of interest for homologous recombination were amplified from T. ethanolicus by PCR. The PCR products and the digested backbone plasmid were assembled by Gibson Assembly. Maps for plasmids used to create the $t d k$ deletion in the wildtype, lactate dehydrogenase $(l d h)$ deletion in X20 strain, and $t d k$ integration in X20 strain are shown in Fig. 1b-d. Complete plasmid sequences were deposited in GenBank with accession numbers KU597226, KU597227, KU597228, and KU597229.

\section{Gene deletion and integration}

To create a background strain for negative selection, gene deletion PCR products from plasmid pTE_tdk were amplified with primers p1 and p2 from Gibson Assembly mixture, column purified, and transformed into T. ethanolicus wild-type strain. Colonies were picked on FUDR agar plates for colony PCR using primers $\mathrm{p} 4$ and $\mathrm{p} 7 \mathrm{fol}$ lowed by gel confirmation.

The gene lactate dehydrogenase $(l d h)$ was deleted on the $t d k$ deletion background strain. PCR products from plasmid pTE_ldh were amplified with primers $\mathrm{p} 1$ and $\mathrm{p} 2$ from Gibson Assembly mixture, column purified, and transformed into T. ethanolicus. Colonies were picked on kanamycin agar plates for colony PCR using primers $\mathrm{p} 8$ and p13 followed by gel confirmation. A verified colony was chosen and plated on FUDR plates. Colonies were picked for colony PCR using primers p20 and p 21 followed by gel confirmation.

The $t d k$ gene was inserted back into the background strain with $t d k$ deletion. Because there are two overlapping downstream regions of the $t d k$ gene in plasmid PTE_tdk(com), integration PCR products could not be amplified with primers p1 and p2. Thus, Gibson Assembly mixture was transformed into DH5 $\alpha$ competent $E$. coli (\#C2987H, NEB) and plasmid was isolated for transformation. Colonies were picked on kanamycin agar plate for colony PCR using primers p14 and p3 followed by gel confirmation. A verified colony was chosen and plated on FUDR $5 \mathrm{mg} / \mathrm{L}$ or FUDR $0 \mathrm{mg} / \mathrm{L}$ plates. Colonies were picked for colony PCR using primers p18 and p19 followed by gel confirmation. Four colonies were chosen for additional confirmation by resequencing. 
Table 2 List of primers used

\begin{tabular}{ll}
\hline Name & Sequence \\
\hline p1 & GAGAAAGGCGGACAGGTA \\
p2 & AATTCTCTTACTGTCATGCC \\
p3 & TGCCTCCTCATCCTCTTC \\
p4, p14 & TTAACCTATAAAAATAGGCGTATCACGAGATGCATCAGCGCCCTGAAGAAGTAACTGACA \\
p5 & CCACCTATATCGGTTTTCTTCATCTCTACACCTCTTTTAGTCTTCACCACTCTAACCCCC \\
p6 & AAAAGAGGTGTAGAGATGAAGAAAACC \\
p7, p17 & CTCCCCGCGCGTTGGCCGATTCATTAATGATGCATCAGGCAGTTCCGCTTCAAGTTTAGG \\
p8 & GACATTAACCTATAAAAATAGGCGTATCACGAGATGCATCAGTGCCGCCTTGTACAGTTT \\
p9 & TTTTCACACTGTGACTTTTTATATGCAAAAAAGAGGTTTCCACCGACAAATCCAGAGCC \\
p10 & GGAAACCCTCTTTTTTGCAT \\
p11 & ATCTTACCTATCACCTCAAATGGTTCGCTGGGTTTTACGCAGCGAACTTCCCGTAGCAAT \\
p12 & CTAATCTTTTCTGAAGTACATCCGCAACTGTCCATACTCCAGTTGATATAAGCCACGGGG \\
p13 & CCGCGCGTTGGCCGATTCATTAATGATGCATCAGCCTCTTATATGTCAAGCTCTTGTATT \\
p15 & TACCTATCACCTCAAATGGTTCGCTGGGTTTACGCAGGCAGTTCCGCTTCAAGTTTAGG \\
p16 & TCTGAAGTACATCCGCAACTGTCCATACTCCAGAAAAGAGGTGAGAGATGAAGAAAACC \\
p18 & AGAAGAATTGGAGGCCAT \\
p19 & CTGCAGAATAAGTAAGGCT \\
p20 & TGCCGCCTTGTACAGTTT (binding sites the same as p8) \\
\hline
\end{tabular}

Note: primer-binding regions are highlighted in italic fonts

\section{Transformation and mutant selection}

Transformation was performed via a natural competence protocol as described previously [9]. For positive selection of mutants with DNA integration onto genome by homologous recombination, up to $250 \mu$ l transformed culture was mixed with $20 \mathrm{~mL}$ CTFUD medium with $0.8 \%(\mathrm{w} / \mathrm{v})$ agar supplemented with $400 \mathrm{mg} / \mathrm{L}$ kanamycin sulfate (\#420311, EMD Millipore), poured into a Falcon $100 \mathrm{~mm}$ petri dish (\#351029, Corning), and then incubated at $65{ }^{\circ} \mathrm{C}$ after solidification. For negative selection to create the background strain X20 or to the remove antibiotic marker by homologous recombination, a confirmed colony from the positive selection step was diluted in $50 \mu \mathrm{L}$ DI water. Up to $25 \mu \mathrm{L}$ diluent was mixed with $5 \mathrm{~mL}$ CTFUD medium with $0.8 \%$ (w/v) agar supplemented with $50 \mathrm{mg} / \mathrm{L}$ 5-fluoro-2'-deoxyuridine (FUDR) (\#F0503, Sigma), poured into a $100 \mathrm{~mm}$ Quad-petri dish (\#70686, Electron Microscopy Sciences), and then incubated at $65^{\circ} \mathrm{C}$ after solidification. For each selection step, seven colonies were picked 2-3 days after incubation for colony PCR followed by gel confirmation.

\section{Measurement of fermentation profile}

The wild-type strain together with the engineered strains X20, X32, and X20C were cultured in CTFUD medium with cellobiose at an initial concentration of $4.25 \mathrm{~g} / \mathrm{L}$ in Corning culture tubes (\#430157) with 5-mL reaction volume. Inoculum prepared in the same medium was added at $10 \%(\mathrm{v} / \mathrm{v})$ inoculation. The tubes were incubated at $65{ }^{\circ} \mathrm{C}$ in an anaerobic chamber for 1 day. Cell growth was measured intermittently via optical density at $600 \mathrm{~nm}$ with a WPA Biowave cell density meter (CO8000, Cambridge, U.K.). Samples were taken at the end for measurement of fermentation products using HPLC. Specific growth rate $(\mu)$ was calculated by exponential curve fitting of optical density as a function of time. Ethanol yield $\left(Y_{\text {eth }}\right)$ was calculated by Eq. (1) and carbon recovery $\left(R_{\mathrm{c}}\right)$ was calculated by Eq. (2).

$$
\begin{aligned}
& Y_{\mathrm{eth}}=\frac{[\text { Ethanol }]}{1.053 \times[\text { Cellobiose }]} \\
& R_{\mathrm{c}}=\frac{[\text { Lactate }]+1.5 \times[\text { Acetate }]+1.957 \times[\text { Ethanol }]}{1.053 \times[\text { Cellobiose }]}
\end{aligned}
$$

\section{Analytical methods}

PCR amplification and colony PCR were both performed with Phusion High-Fidelity PCR Master Mix with HF Buffer (\#M0531L, NEB). Plasmid was extracted using QIAprep Spin Miniprep Kit (\#27106, Qiagen). Backbone plasmid digestion was performed with PvuII-HF (\#R3151S, NEB) in CutSmart Buffer (\#B7204S, NEB) at $50{ }^{\circ} \mathrm{C}$ for $15 \mathrm{~min}$. Gel purification was performed on $1 \%$ agarose gel with $0.01 \%(\mathrm{v} / \mathrm{v})$ SYBR Safe DNA Gel Stain florescent indicator (\#S33102, Thermo Fisher Scientific) and recovered using Zymoclean Gel DNA Recovery Kit 
(\#D4002, Zymo Research). Plasmids were constructed with Gibson Assembly Master Mix (\#E2611L, NEB) at $50{ }^{\circ} \mathrm{C}$ for $1 \mathrm{~h}$. Gels were run on Owl Easy Cast Mini Gel Electrophoresis Systems (\#B1A, Thermo Scientific) for 35-40 min at 120 volts with Owl Compact Power Supply (\#EC300XL, Thermo Scientific) and imaged on a Molecular Imager Gel Doc XR + System (\#1708195EDU, Bio-Rad). Fermentation metabolite concentrations were determined using a Waters HPLC system equipped with an Aminex HPX-87H column (Bio-Rad Laboratories, Hercules, CA) operated at $60{ }^{\circ} \mathrm{C}$. The mobile phase was $5 \mathrm{mM} \mathrm{H}_{2} \mathrm{SO}_{4}$ at a flow rate of $0.6 \mathrm{~mL} / \mathrm{min}$.

\section{Abbreviations}

$t d k$ : thymidine kinase gene; Tdk: thymidine kinase protein; $h t k$ : high-temperature kanamycin resistance gene; /dh: lactate dehydrogenase gene; FUDR: 5-fluoro-2'-deoxyuridine; hpt: hypoxanthine phosphoribosyl transferase gene; Hpt: hypoxanthine phosphoribosyl transferase protein; F-dUMP: fluoro-dUMP; ThyA: thymidylate synthase protein; WT: wild-type.
\end{abstract}

\section{Authors' contributions}

XS constructed pZJ24htktdk, pTE_tdk, pTE_ldh, and pTE_tdk(com), and performed gene deletion and integration and the characterization. JZ constructed pZJ24 and participated in the design of this study. DO and LL participated in the design of this study. All authors have revised the paper critically for intellectual content. All authors have read and approved the final manuscript.

\section{Author details}

${ }^{1} 14$ Engineering Drive, Thayer School of Engineering, Dartmouth College, Hanover, NH 03755, USA. ${ }^{2}$ DOE BioEnergy Science Center, Oak Ridge National Laboratory, Oak Ridge, TN 37831, USA.

\section{Acknowledgements}

The authors were supported by the BioEnergy Science Center (BESC), a U.S Department of Energy (DOE) Research Center supported by the Office of Biological and Environmental Research in the DOE Office of Science, Oak Ridge National Laboratory. Oak Ridge National Laboratory is managed by University of Tennessee UT-Battelle LLC for the Department of Energy under Contract No. DE-AC05-00OR22725.

\section{Competing interests}

Professor Lynd is an equity holder in Enchi Corp., a biofuel start-up. The authors declared that they have no competing interests.

Received: 1 February 2016 Accepted: 22 April 2016

Published online: 04 May 2016

\section{References}

1. Lynd LR, Wyman CE, Gerngross TU. Biocommodity Engineering. Biotechnol Prog. 1999;15:777-93.

2. Lynd LR, Jin H, Michaels JD, Wyman CE, Dale B. Bioenergy: background, potential, and policy. Washington: Center for Strategic and International Studies; 2003.
3. Wiegel J, Ljungdahl L. Thermoanaerobacter ethanolicus gen. nov., spec. nov., a new, extreme thermophilic, anaerobic bacterium. Arch Microbiol. 1981;128:343-8.

4. Hild HM, Stuckey DC, Leak DJ. Effect of nutrient limitation on product formation during continuous fermentation of xylose with Thermoanaerobacter ethanolicus JW200 Fe(7). Appl Microbiol Biotechnol. 2003:60:679-86.

5. Lynd LR, Weimer PJ, van ZyI WH, Pretorius IS. Microbial cellulose utilization: fundamentals and biotechnology. Microbiol Mol Biol Rev. 2002;66:506-677.

6. Lynd LR, van Zyl WH, McBride JE, Laser M. Consolidated bioprocessing of cellulosic biomass: an update. Curr Opin Biotechnol. 2005;16:577-83.

7. Taylor MP, Eley KL, Martin S, Tuffin MI, Burton SG, Cowan DA. Thermophilic ethanologenesis: future prospects for second-generation bioethanol production. Trends Biotechnol. 2009;27:398-405.

8. Peng H, Fu B, Mao Z, Shao W. Electrotransformation of Thermoanaerobacter ethanolicus JW200. Biotechnol Lett. 2006;28:1913-7.

9. Shaw AJ, Hogsett DA, Lynd LR. Natural competence in Thermoanaerobacter and Thermoanaerobacterium species. Appl Environ Microbiol. 2010;76:4713-9.

10. Shaw AJ, Covalla SF, Hogsett DA, Herring CD. Marker removal system for Thermoanaerobacterium saccharolyticum and development of a markerless ethanologen. Appl Environ Microbiol. 2011;77:2534-6.

11. Argyros D, Tripathi S, Barrett T, Rogers S, Feinberg L, Olson D, Foden J, Miller B, Lynd L, Hogsett D, Caiazza N. High ethanol titers from cellulose by using metabolically engineered thermophilic, anaerobic microbes. Appl Environ Microbiol. 2011;77:8288-94.

12. Pritchett $M$, Zhang J, MetcalfW. Development of a markerless genetic exchange method for Methanosarcina acetivorans C2A and its use in construction of new genetic tools for methanogenic archaea. Appl Environ Microbiol. 2004;70:1425-33.

13. Sivakumar S, Porter-Goff M, Patel PK, Benoit K, Rhind N. In vivo labeling of fission yeast DNA with thymidine and thymidine analogs. Methods. 2004;33:213-9.

14. Nucleotide metabolism [http://themedicalbiochemistrypage.org/nucleotide-metabolism.php].

15. Czakó M, Márton L. The herpes simplex virus thymidine kinase gene as a conditional negative-selection marker gene in Arabidopsis thaliana. Plant Physiol. 1994;104:1067-71.

16. Gardiner D, Howlett B. Negative selection using thymidine kinase increases the efficiency of recovery of transformants with targeted genes in the filamentous fungus Leptosphaeria maculans. Curr Genet. 2004;45:249-55.

17. Khang C, Park S, Lee Y, Kang S. A dual selection based, targeted gene replacement tool for Magnaporthe grisea and Fusarium oxysporum. Fungal Genet Biol. 2005:42:483-92.

18. Hashimoto Y, Yano T, Kuramitsu S, Kagamiyama H. Disruption of Thermus thermophilus genes by homologous recombination using a thermostable kanamycin-resistant marker. FEBS Lett. 2001;506:231-4.

19. Olson DG, Lynd LR. Transformation of Clostridium thermocellum by electroporation. In: Gilbert HJ, editor. Methods in enzymology, vol. 510. Amsterdam: Elsevier Inc; 2012. p. 317-30.

20. Zhou J, Olson DG, Lanahan AA, Tian L, Murphy SJL, Lo J. Physiological roles of pyruvate ferredoxin oxidoreductase and pyruvate formate-lyase in Thermoanaerobacterium saccharolyticum JW/SL-YS485. Biotechnol Biofuels. 2015;8:138

21. Hoseki J, Yano T, Koyama Y, Kuramitsu S, Kagamiyama H. Directed evolution of thermostable kanamycin-resistance gene: a convenient selection marker for Thermus thermophilus. J Biochem. 1999;126:951-6. 